"WNiF-890197- 3

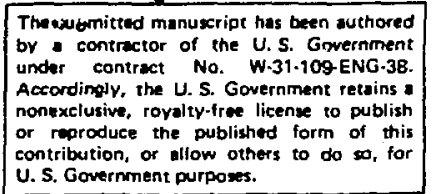

WF- 104

ANL-HEP-CP-89-13

February 13, 1989

\title{
Instability of Compensated Beam-Beam Collisions
}

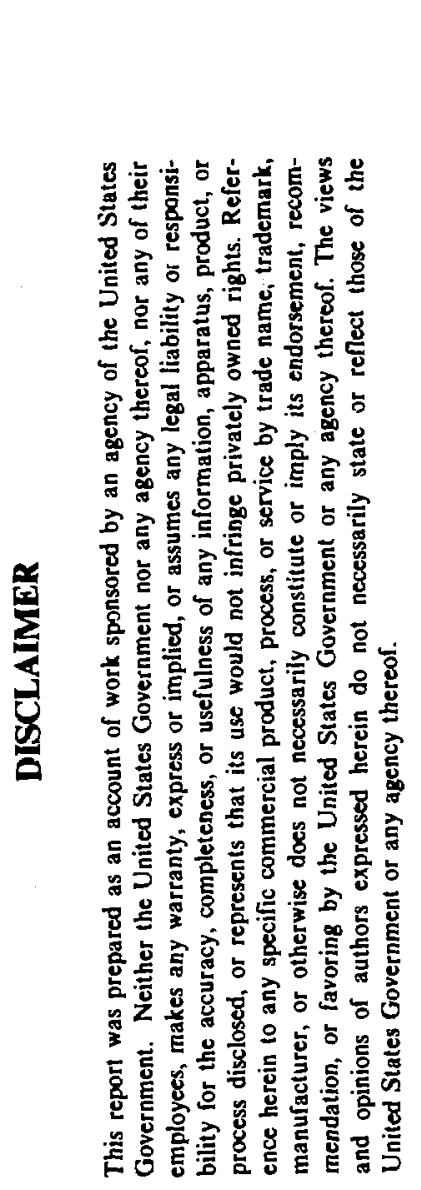

\author{
J. B. ROSENZWEIG* \\ Fermi National Acceierator Laboratory \\ ANL-HEP-CP--89-13 \\ DE89 009818 \\ Batavia, Illinois 60510

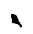 \\ B. AUTIN $^{\dagger}$ \\ Lawrence Berkeley Laboratory \\ University of California \\ Berkeley, $C A$ 9\$720
}

and

PISIN CHEN

Stanford Linear Accelerator Center

Stanford, CA 94905

\begin{abstract}
Submitted to the
1989 Lake Arrowhead Workshop on Advanced Accelerator Concepts
\end{abstract}

*Also at Argonne National Laboratory.

tPermanent address: CERN, Geneva, Switzerland.

Work supported by the U.S. Department of Energy, Division of High Energy

Physics, Contract W-31-109-ENG-38. 


\section{Introduction}

As recent discussions have pointed out, the beam-beam disruption phenomena in linear colliders are increasingly seen as a source of serious problems for these machines. The maximum disruption-induced angle introduces a constraint on final focusing system apertures, which has led to the complication of interaction point crossing angles. Additional constraints arise due to the desire to minimize the energy spread due to beamstrahlung energy loss to acceptable levels. Much more seriously, the beamstrahlung photons produced during disruption have been shown, in a recent calculation by $\mathrm{P}$. Chen, ${ }^{[1]}$ to pair-produce via interaction with the opposing bunch's disruption fields. This is a source of potentially serious background problem, as for TLC parameters Chen predicts more than $10^{6}$ lower energy $e^{+} e^{-}$pairs created during each collision. These pairs have a broad energy spectrum, much of which is susceptible to be scattered at large angles from the beam-beam focusing fields, flooding the detector with spurious events.

All of these problems taken together tend to outweigh the potential gains derived from disruption luminosity enhancement. As this trade-off has been at issue for some time, ways of minimizing the deleterious effects of disruption have been proposed. In particular, a plasma compensation scheme, in which the motion of the plasma electrons in the presence of the colliding beams provides neutralizing charge and current densities, has been proposed and studied. ${ }^{[2]}$ As a very large plasma density is required for compensation, this scheme introduces a large number of background beam-ion events. Channell proposes an interesting variation on this theme, in which a positronium plasma is created at the interaction point, ${ }^{|3|}$ thus avoiding the beam-ion backgrounds. Unfortunately, presently conceivable methods for creation of the necessary positronium plasma do not provide high enough densities for compensation. In fact, a likely candidate for such a method would be to rely on pair production from the beamstrahlung photons. Since this scenario takes us full circle to our original motivation for this work, we 
can see immediately that this scheme will not provide compensation for a TLC -like machine. Thus we are driven to examine other options for compensation of intense colliding beams.

A natural alternative for full compensation is to consider the overlapping of nearly identical high energy $e^{+}$and $e^{-}$bunches, and the collision of two such pairs - in other words, collision of two opposing relativistic positronium plasmas. It should be noticed that while the luminosity for all collisions is increased by a factor of four in this scheme, the event rate for $e^{+} e^{-}$collisions is only increased by a factor of two. The other factor of two corresponds to the addition of $e^{+} e^{+}$and $e^{-} e^{-}$collisions to the interaction point. This beam compensation scheme, which has been examined through computer simulation by Balakin and Solyak $^{|4,5|}$ in the Soviet Union, promises full neutralization of beam charges and currents. These numerical investigations have shown that plasma instabilities exist in this nominally neutral system. Although the implementation of this idea seems technically daunting, the potential benefits (beamstrahlung and disruption suppression, relaxation of final focus system constraints) are such that we should consider the physics of these collisions further. In the remainder of this paper, we theoretically analyze the issues of stability and bunch parameter tolerances in this scheme.

This four-beam system we have described is assumed to be nearly charge and current neutral, in order to provide the compensation we seek. The instabilities that grow during the collision of these paired beams are due to the amplification of initial imperfections in the neutrality of the system. These imperfections can take a variety of forms: misalignment of the propagation axes of the comoving $e^{+}$ and $e^{-}$beam, mismatch of their relative charge, shape, and longitudinal position. The problem can be split into two classes, the first being amplification of the dipole fields due to transverse misalignment of the comoving beams. This mode is related to the kink instability, which is well known in the context of plasma and beam physics. The imperfections of the other class, consisting of mismatches in quantities other than transverse alignment, contribute mainly to a 'quadrupole' 
or focusing instability.

As the assumed purpose of this work is to minimize the effects of beam-beam disruption, we consider the limit of flat beam collisions. Operation in this regime serves to suppress disruption and beamstrahlung effects even in the absence of compensation. The generalization of our results to the inherently more disruptive round beam case is discussed in a subsequent section.

\section{The Dipole Instability}

We begin by analyzing the case of the dipole instability, in which an initial transverse separation of the charge distributions in one comoving $e^{ \pm}$beam pair causes a separation in the oncoming pair. The separation in the oncoming pair then amplifies the dipole strength of the charge separation in the originally misaligned beam pair. Thus an instability of the relative $e^{ \pm}$beam positions results. To obtain the growth rate for the dipole instability we examine the following model system: two pairs of slab $e^{+}$and $e^{-}$beams of energy $\gamma m_{e} c^{2}$ and uniform density $n_{b}$, infinite in longitudinal (propagation) direction $z$, and much larger in the $x$ than in the $y$ dimension, where the beam has vertical height $b$. We examine the collective motion of the beam slabs assuming a small initial misalignment in $y$ of both beam pairs. We take the right-going $e^{+}$beam to be vertically misaligned from the $e^{-}$beam before collision by $\delta_{1}$, and the left-going $e^{+}$and $e^{-}$beams misaligned by $\delta_{2}$. We also assume that the beam pairs have a small misalignment $\delta_{0}$ with respect to each other. If the misalignments are small, $\delta_{0,1,2} \ll b$, then the net vertical dipole forces felt by the bulk of the $e^{+}$beams due to the excess exposed charge on the oncoming beam edges are

$$
F_{1,2}=-\left(1+\beta_{b}^{2}\right) 4 \pi e^{2} n_{b} \delta_{2,1} \simeq-8 \pi e^{2} n_{b} \delta_{2,1}
$$

where $\beta_{b}=v_{b} / c$ is the normalized beam velocity and $n_{b}$ is the beam density, assumed nearly constant within the bunch boundaries. The subscript 1 and 2 on 
the force refers to that felt by the right-going and left-going positron bunches, respectively, and opposite sign forces are felt by the comoving electron beams.

Following the method employed by Chin ${ }^{[6]}$ (the 'two-string' model ${ }^{[7]}$ ) in examining the kink instability for uncompensated beam-beam collisions, we write equations of motion for the vertical positions $y^{ \pm}$of the $e^{ \pm}$beams

$$
\left(\frac{\partial}{\partial t} \pm v_{b} \frac{\partial}{\partial z}\right)^{2} y_{1,2}^{+}=-\frac{\omega_{b}^{2}}{2} \delta_{2,1}
$$

and

$$
\left(\frac{\partial}{\partial t} \pm v_{b} \frac{\partial}{\partial z}\right)^{2} y_{1,2}^{-}=\frac{\omega_{b}^{2}}{2} \delta_{2,1}
$$

where

$$
\omega_{b}^{2}=\frac{16 \pi e^{2} n_{b}}{\gamma m_{c}},
$$

and we have ignored the transverse component of the convective derivative. This system can be simplified by rewriting it in terms of the misalignments $\delta_{1,2}$ as follows:

$$
\left(\frac{\partial}{\partial t} \pm v_{b} \frac{\partial}{\partial z}\right)^{2} \delta_{1,2}=-\omega_{b}^{2} \delta_{2,1}
$$

If we assume a dependence of $\exp (i k z-i \omega t)$ for the unknown quantities in Eqs. (5), and require that the resulting algebraic system have a non-trivial solution, we obtain the dispersion relation

$$
\omega^{2}=\left(k v_{b}\right)^{2}-\omega_{b}^{2}
$$

This system is absolutely unstable for all $k$, and has a maximally unstable mode occuring at $k=0$ with a growth rate $\nu=-i \omega=\omega_{b}$.

To explicitly illustrate the physical behavior of this mode, we rewrite Eqs. (5) with the convective derivative suppressed. Defining new dependent variables 
by

$$
\psi_{1,2}=\delta_{1} \pm \delta_{2}
$$

the equations of motion transform to

$$
\frac{d^{2} \psi_{1,2}}{d t^{2}}= \pm \omega_{b}^{2} \psi_{1,2}
$$

The solution for $\psi_{2}$ is oscillatory with frequency $\omega_{b}$ while the solution for $\psi_{1}$ is an exponential, with a growth rate also given by $\omega_{b}$. As an example of a purely growing disturbance, we take the initial misalignments to be equal $\delta_{1,2}(t=0)=$ yo with no initial transverse motion, and write

$$
\delta_{1,2}=y_{0} \exp \left(\omega_{b} t\right)
$$

Physically, what is happening is that the electrons from one beam are attracted by the oncoming positrons and repulsed by the oncoming electrons, and vice versa for the comoving positrons. Thus the comoving beams split and diverge exponentially until the misalignments are no longer small compared to the bunch height. We are not at present interested in the large amplitude behavior of the instability, however, as this corresponds to a limit where nearly all of the compensation has been lost, with a concommitant return of disruption and beamstrahlung problems.

At first glance, the physics of the four-beam collision we have considered here is quite a tit different from the two-beam $e^{ \pm}$results. It is straightforward to show that the different behavior is due to the presence of like sign species that repulse each other. In his instability analysis of the two-beam $e^{ \pm}$kink instability, Chin finds the dispersion relation

$$
\omega_{b}^{2}\left[\left(\omega+k v_{b}\right)^{2}+\left(\omega-k v_{b}\right)^{2}\right]=2\left(\omega^{2}-\left(k v_{b}\right)^{2}\right)^{2}
$$

The maximum growth rate associated with this relation is $\nu=\omega_{p} / \sqrt{8}$, occuring at $k=\omega_{p} / v_{b}$. The kink instability occurs when the two misaligned colliding beams 
are attracted to each other, but overshoot as they chase each other, resulting in a growing disturbance at the 'natural' plasma wave-number $k=\omega_{p} / v_{b}$. If one repeats the analysis for like sign (repulsing) beams colliding, however, the dispersion relation is now

$$
\omega_{b}^{2}\left[\left(\omega+k v_{b}\right)^{2}+\left(\omega-k v_{b}\right)^{2}\right]=-2\left(\omega^{2}-\left(k v_{b}\right)^{2}\right)^{2},
$$

and the maximum growth rate $\nu=\omega_{b}$ occurs, again, at $k=0$. This illustrates the feature that when the two effects of attraction and repulsion driven instability are present, as in the four-beam collision, the repulsion dominates the growth of the disturbance.

We now apply this result to a flat beam linear collider case. The previous analysis does not take into account the variations of the beam charge density as a function of position in the bunch, the effects of the transient nature of the collision, or of finite beam emittance. In spite of these shortcomings, the analysis can give a reasonable estimate on the cumulative growth of the instability during collision. For flat, tri-Gaussian bunches, the beam density is given by

$$
n_{b}=-\frac{N}{(2 \pi)^{3 / 2} \sigma_{x} \sigma_{y} \sigma_{z}},
$$

and the associated definition of vertical disruption parameter is

$$
D_{y}=\frac{2 N r_{e} \sigma_{z}}{\gamma \sigma_{x} \sigma_{y}} .
$$

As most of the beam charge per bunch is located in a region of length $2 \sigma_{z}$, the interaction time for the instability is approximately $\tau=\sigma_{z} / c$. the final amplitide of the misalignment $\delta_{f}$ accumulated during the collision can be estimated by setting in Eq. (9),

$$
\delta_{f}=y_{0} \exp \left(\omega_{b} \sigma_{z} / c\right)=y_{0} \exp \left[\left(4 D_{y} / \sqrt{2 \pi}\right)^{1 / 2}\right]
$$

Thus the condition for one e-folding of the instability is approximately $D_{y}=0.63$. This should be considered a pessimistic estimate, however, as all the phenomena 
that we have ignored in this analysis - in particular, variation of beam densities during the collision, and thermal effects - will tend to diminish or stabilize the instability growth. One can conclude with reasonable certainty, however, that disruption parameters of $D_{y}>5$ lead to severe dipole instability problems.

\section{The Quadrupole Instability}

A focusing or instability can occur when the densities of the comoving $e^{ \pm}$pair are not identically matched, for reasons of unequal total beam charge, radius, or arrival time at the interaction point. We thus need to write equations of motion for the beam densities involved. We begin, as wich the dipole instability, by writing the force equations for the bearn fluids, where we make the identification

$$
\mathbf{F}_{1,2}=\gamma m_{e}\left(\frac{\partial}{\partial t} \pm v_{b} \frac{\partial}{\partial z}\right) \mathrm{v}
$$

The transverse disruption forces due to net charge density in the oncoming beam pair are approximately equal to twice the electric forces, and thus, for the rightgoing $e^{ \pm}$beam pair we have

$$
\nabla \cdot \mathbf{F}_{1}= \pm \nabla \cdot \mathbf{E}_{2}= \pm 8 \pi e^{2}\left(n_{2}^{+}-n_{2}^{-}\right)
$$

where $n^{ \pm}$refer to the $e^{ \pm}$beam densities. An analogous equation holds for the left-going beam pair. If we assume that all densities are nearly equal to a nominal density $n_{b}$, then the linearized equation of continuity gives, for all species,

$$
n_{b} \nabla \cdot \mathrm{v}=-\frac{\partial n}{\partial t}
$$

Combining Eqs. (16) and (17), we obtain the fluid equations

$$
\left(\frac{\partial}{\partial t} \pm v_{b} \frac{\partial}{\partial z}\right) \frac{\partial n_{1,2}^{+}}{\partial t}=\frac{\omega_{b}^{2}}{2}\left(n_{2,1}^{+}-n_{2,1}^{-}\right)
$$


and

$$
\left(\frac{\partial}{\partial t} \pm v_{b} \frac{\partial}{\partial z}\right) \frac{\partial n_{1,2}^{-}}{\partial t}=-\frac{\omega_{b}^{2}}{2}\left(n_{2,1}^{+}-n_{2,1}^{-}\right)
$$

Introducing the net charge density inside a beam pair $\Delta n_{1,2} \equiv n_{1,2}^{+}-n_{1,2}^{-}$for new dependent variables, Eqs. (18) and (19) become

$$
\left(\frac{\partial}{\partial t} \pm v_{b} \frac{\partial}{\partial z}\right) \frac{\partial \Delta n_{1,2}}{\partial t}=-\omega_{b}^{2} \Delta n_{2,1}
$$

The dispersion relation obtained from Eq. (20) is

$$
\omega^{2}\left(\omega^{2}-\left(k v_{b}\right)^{2}\right)=\omega_{b}^{4}
$$

The maximum growth rate for an unstable mode is once again found at $k=0$, where we have $\nu=\omega_{b}$, which is identical to the dipole instability growth rate. This result can be verified from Solyak's previous numerical work. ${ }^{[4 \mid}$ The growih rates for the dipole and quadrupole instability in these simulations appear to be nearly equal.

The fact that the dipole and quadrupole instabilities have the same wave number and growth rate is not surprising, as their physical mechanisms are nearly the same. In the quadrupole instability, a bunch with excess density focuses the oncoming beam of opposite polarity, while defocusing the oncoming beani of the same polarity. The focusing feedback from the oncoming charge density then allows for exponential growth of the mode. 


\section{Lower Energy Beam Compensation}

If one is to implement a beam-based compensation scheme, then it is reasonable to ask if lower energy beams are useful for providing the compensating charge and current density. Despite the obvious advantages such a scheme would have, it should be noted that this is a problematic suggestion from a technical stand-point, as one is focusing very low emittance high-energy $\gamma_{1} m c^{2}$ beams to very small $\beta$-functions. To achieve the same beam dimensions and densities using lower energy $\gamma_{2} m c^{2}$ beams, which will naturally have larger emittances by a factor of $\gamma_{1} / \gamma_{2}$ seems quite dificult. In addition, as Solyak determined from simulation results, the instabilities have an even higher growth rate if two of the four beams are of lower energy. ${ }^{[4]}$

In order to quantify the relative instability growth rate in this regime we take the case where the colliding high energy $e^{-} e^{+}$beams are compensated by low energy $e^{+} e^{-}$beams. Defining two new beam plasma frequencies by

$$
\omega_{1,2}^{2}=\frac{16 \pi e^{2} n_{b}}{\gamma_{1,2} m}
$$

we examine the dipole instability fluid equations, which can be written

$$
\left(\frac{\partial}{\partial t} \pm v_{b} \frac{\partial}{\partial z}\right)^{2} \delta_{1,2}=-\frac{1}{2}\left(\omega_{1,2}^{2}+\omega_{2,1}^{2}\right) \delta_{2,1}
$$

From these equations we obtain the dispersion relation

$$
\omega^{2}=\left(k v_{b}\right)^{2}-\frac{1}{2}\left(\omega_{1}^{2}+\omega_{2}^{2}\right)
$$

This system is again absolutely unstable for all $k$, with a maximally unstable mode of growth rate given by $\nu^{2} \simeq(1 / 2)\left(\omega_{1}^{2}+\omega_{2}^{2}\right)$ occuring at $k=0$. If $\gamma_{1} \gg \gamma_{2}$, the growth rate of the instability is $\nu \simeq \omega_{2} / \sqrt{2}$, or about a factor of $\sqrt{\gamma_{1} / 2 \gamma_{2}}$ larger than for equal energy compensation. It is clear that the lack of rigidity in the trajectory of the lower ener gy beams allows for larger instability growth rate. 
The instability of this four-beam system can be shown, as before in the equal energy case, to be dominated by the repulsion of the like sign species colliding. It is interesting to note that in the equivalent uncompensated two-beam case, e.g. high energy $e^{-}$beams on low energy $e^{+}$beams, that the kink instability is greatly diminished. This is because the two oscillators (beams) must have nearly the same frequency for their attracting interaction to become violently unstable. This observation is relevant to the conceptual design of B-factories, as the physics of detecting $B$ meson decays requires asymmetric beam energies. The high luminosity needed for B-factories implies that the disruption parameter will be large, $D \sim 10-30$, so an improvement in the stability of the collisions is in fact quite desirable. In this scenario one might envision taking full advantage of disruption-induced luminosity enhancement without the worrying about accompanying instability problems.

\section{Thermal Effects}

The beam-beam instability growth rates calculated above are based on a cold fluid model. The transverse temperature of the beams can diminish or eliminate the growth of the unstable modes considered here, as the random motion of particles can lead to dissipation of the fluctuations in net beam density that give rise to instabilities. A physical criterion for this to happen in the dipole mode is when a particle with the rms transverse velocity associated with the beam distribution can traverse the initial oncoming misalignment $y_{0}$ in less than a growth time $\omega_{b}^{-1}$. This stability condition can be formulated as

$$
A^{2}>D_{y}\left(\frac{y_{0}}{\sigma_{y}}\right)
$$

where $A=\sigma_{z} / \beta_{y}^{*}$ is the ratio of the rms beam length to the vertical $\beta$-function at the interaction point. This quantity is a measure of a depth of focus problem; if $A>1$, then the luminosity will be degraded due to the transverse spreading 
of the bearns during the interaction. Recently proposed TLC conceptual designs have chose values of $A$ slightly less than unity. ${ }^{|0|}$

A similar criterion can be derived for the quadrupole instability. If we require that the remnant focusing strength due to an excess oncoming charge density $\pm \Delta n_{0}$ be insufficient to confine the comoving $e^{--}\left(e^{+}\right)$beam, then instability will be suppressed. This condition can be stated as

$$
A^{2}>D_{y}\left(\frac{\Delta n_{0}}{n_{b}}\right)
$$

Due to depth of focus considerations, we take the maximum value of $A=1$ to derive tolerances on the initial imperfections in compensation. We thus have $\left(\frac{\Delta n_{0}}{n_{b}}\right)<D_{y}^{-1}$ and $\left(\frac{y_{0}}{\sigma_{y}}\right)<D_{y}^{-1}$ for thermal stabilization of the quadrupole and dipole instäbilities, respectively. Threse tolerances should be compared to those derived from acceptable levels of beamstrahlung, which we discuss below.

\section{Tolerances}

The tolerances to which one must match the $e^{+}$and $e^{-}$beam disiributions can be easily estimated for misalignments by using Eq. (1), and working through the expected level of beamstrahlung with its related problems. The effective magnetic dipole field strength encountered due to misalignments is given by $B_{e f f} \simeq 8 \pi e n_{b} y_{0}$. We now define an effective beamstrahlung parameter $\Upsilon_{\text {eff }}=\gamma B_{\text {eff }} / B_{c}$, where $B_{c}=m^{2} c^{3} / e \hbar$ is the Schwinger critical field. Recent calculations have shown that for $\Upsilon \leq 1$, the problem of coherent pair creation is suppressed. Explicitly, this requires that

$$
\frac{y_{0}}{\sigma_{y}}<\frac{\sigma_{z} \sigma_{z}}{4(2 \pi)^{3 / 2} N \gamma \lambda_{c} r_{e}}
$$

where $\lambda_{c}$ is the electron Compton wavelength. 
Since the average effective field in the case of unmatched densities is $B_{\text {eff }} \simeq$ $4 \pi e \Delta n_{0} \sigma_{y}$, the tolerance requirement on density matching for suppressing beamstrahlung pair production can be similarly stated as

$$
\frac{\Delta n_{0}}{n_{b}}<\frac{\sigma_{x} \sigma_{z}}{2(2 \pi)^{3 / 2} N \gamma \lambda_{c} r_{e}}
$$

For $\mathrm{TeV}$ colliders, one would expect these tolerances to be on the order of ten percent, given the potential severicy of the disruption effects. This appears to be more challenging than uncompensated design tolerances. For TLC design, alignment precision implied is already on the sub-nanometer scale - alignment would undoubtedly have to be even better if we rely on compensation, and in addition one must also accomplish this with four beams simultaneousiy. If one keeps the luminosity constant, however, the relative alignment precision necessary is lessened considerably if round beams are employed. We now consider the feasibility of this option.

\section{Round Beams}

It is easy to show that the superposition of two slightly misaligned round electron and positron beams also gives rise to a dipole field, of strength $B_{\text {eff }} \simeq$ $4 \pi e n_{b} y_{0}$, over most of the beam distribution: Note that this is one-half the dipole strength of the flat beam case if one keeps the luminosity and misalignment constant, and that it scales only with the absolute, not relative, value of the misalignment. From this point of view, there to be some slight advantage to using round beams in a compensated collision scheme.

On the other hand, a relative error in density matching is very severe in the round beam limit. The effective average field strength due to this error is given by $B_{e f f} \simeq 2 \pi e \Delta n_{0} \sigma_{r}$, which, for a given density error, is greater than a fiat beam case with $R=\sigma_{x} / \sigma_{y}$ by approximately a factor of $(R+1) / 2$. Thus it is

apparent that flat beams have more tolerance to this scheme, as one has already 
made efforts to minimize the potential beamstrahlung effects. Conversely, round beams are very unforgiving of density mismatches, as the uncompensated $\Upsilon$ is much larger than the equivalent luminosity flat beam case.

\section{Discussion}

In the design of linear colliders, there are two parameters which globally measure the strength of the beam-beam interaction - the disruption parameter $D_{y}$ and the beamstrahlung parameter $\Upsilon$. One would like to make both of these numbers smaller than approximately unity, due to the problems associated with induced energy spread, fluid instabilities, and coherent beamstrahlung pair creation. One way to accomplish this is to lower the amount of charge in each pulse, through either raising the linac repetition rate or by multi-bunching. The latter has been suggested as a way to eliminate coherent pair creation in the TLC.

It should be noted that $D_{y}$ and $\Upsilon$ have opposite dependences on two parameters $-\Upsilon \sim \gamma / \sigma_{z}$ and $D_{y} \sim \sigma_{z} / \gamma$. If one multiplies both of these by the implicit $\gamma$ dependence on beam current density dictated by the energy-squared dependence of the luminosity, which for round beams is $\gamma^{2}$ and for flat beams is $\gamma^{2} / R$, then one sees that both parameters scale upwards with energy. The dependence of $\Upsilon$ on $\gamma$ is much more dramatic, and thus there is a trade-off between the two, which can be played by lengthening the bunch. Of course, the maximum value of $\sigma_{z}$ is constrained either by $\beta_{y}^{*}$ or by wake-field considerations. In any case, one can see that the problem of managing the beam-beam effects will become quite difficult at higher energies.

As all the deleterious effects of the beam-beam interaction are due to the presence of large collective fields, compensation of the sources of these fields remains an important question. We have discussed above some of the difficulties associated with beam compensation, and have estimated stability and tolerance limits for this scheme. These results, in conjunction with the previous simulations ${ }^{\text {[1] }}$ and the experimental evidence for multi-pass instabilities arising from this sort 
of interaction in the DCI facility at Orsay ${ }^{[0,10]}$ establish that the physical phenomena we have discussed present a real challenge for implementation of beam compensation.

Since we have identified the problematic aspects of beam compensation it is worth asking whether plasma compensation suffers from similar afflicting instabilities. This subject is beyond the scope of this paper, but preliminary indications from other investigators is that plasma compensation is much more resistant to these instabilities than beam compensation. ${ }^{[1]}$ Thus, although both schemes for compensation have difficulties, they are not qualitatively the same. The necessity of solving the problems associated with beam-beam disruption provides ample motivation for continued investigation, both theoretical and experimental, of both beam and plasma compensation.

This work supported by U.S. Department of Energy, Contracts W-31-109ENG-38, DE-AC03-76SF0098, and DE-AC03-76SF00515. 


\section{REFERENCES}

1. P. Chen, Stanford Preprint SLAC-PUB-4822, Stanford, CA, December 1988.

2. D. Whittum, A. Sessler, J. Stewart and S. Yu, Berkeley Preprint LBL25759, Berkeley, CA, July 1988.

3. P. Channell, presented at the Santa Barbara Conference on Submicron Beams, July, 1988.

4. N.A. Solyak, Novosibirsk Preprint 88-44, Novosibirsk, USSR, 1988.

5. V. E. Balakin and N.A. Solyak, Proc. XIII Intern. Conf. on High Energy Accelerators, Novosibirsk, USSR, 1986.

6. Y.H. Chin, DESY Preprint DESY 87-011, Hamburg, FRG, January 1987.

7. J.G. Linhart, Plasma Physics, North-Holland, Amsterdam, 1960, p. 141.

8. R. B. Palmer, Stanford Preprint SLAC-PUB-4295, Stanford CA, April, 1987.

9. J. Le Duff, M.P. Level, P.C. Marin, E.M. Sommer, H. Zyngier, Proc. $X I$ Intern. Conf. on High Energy Accelerators, CERN (Geneva 1980).

10. C.Pellegrini, A.M. Sessler, Proc. VI Conf. on High Energy Accelerators (Cambridge 1967).

11. B. Autin, private communication. 